Revue d'histoire de l'Amérique française

REYUE D.HISTOIRE DE L'AMÉRIQUE FRANÇAISE

\title{
Problèmes et plaisirs de la biographie
}

\section{Yvan Lamonde}

Volume 54, numéro 1, été 2000

URI : https://id.erudit.org/iderudit/305658ar

DOI : https://doi.org/10.7202/305658ar

Aller au sommaire du numéro

Éditeur(s)

Institut d'histoire de l'Amérique française

ISSN

0035-2357 (imprimé)

1492-1383 (numérique)

Découvrir la revue

Citer ce document

Lamonde, Y. (2000). Problèmes et plaisirs de la biographie. Revue d'histoire de l'Amérique française, 54(1), 89-94. https://doi.org/10.7202/305658ar d'utilisation que vous pouvez consulter en ligne.

https://apropos.erudit.org/fr/usagers/politique-dutilisation/ 


\title{
Problèmes et plaisirs de la biographie
}

YVAN LAMONDE

Département de langue et littérature françaises Université McGill

\begin{abstract}
Cerges dumézil définissait la méthode comme «le chemin une fois J parcouru». Après ma biographie de Louis-Antoine Dessaulles (Fides, 1994) et quelques contributions (Bibaud, Chandonnet, Désaulniers, Dessaulles, Faribault, Odelin, Raymond) au Dictionnaire biographique du Canada, il me semble y avoir cinq aspects de la biographie susceptibles d'alimenter la réflexion des historiens: le problème de la scientificité et de l'aporie inhérente au genre, l'ouverture sur des casproblèmes, la confrontation au récit, la dimension "thérapeutique " ou d'objectivation de la biographie et la prise en compte du lectorat de l'histoire'.
\end{abstract}

\section{UNE APORIE NON RÉSOLUE}

Le problème central soulevé par la biographie historique peut se résumer à une question: quels sont sa situation et son apport sur le plan scientifique, en quoi est-elle un moyen de connaissance scientifique? La question se pose pour la raison toute simple que la biographie porte sur un individu et que le traitement de l'individu en science pose problème.

1. Sur les problèmes méthodologiques de la biographie, un point de départ bibliographique dans Yvan Lamonde, Louis-Antoine Dessaulles, un seigneur libéral et anticlérical (Saint-Laurent, Fides, 1994), 338. 
Le biographe est d'entrée de jeu confronté au fait qu'il écrit sur un individu qu'il voudra situer dans son époque - l'individu dans son présent et le présent dans l'individu - et dont il sera tenté de vouloir généraliser l'expérience. Là est le nœud: en quoi la vie de Dessaulles parle-t-elle de la société canadienne-française entre 1818 et 1895, de quoi Dessaulles peut-il bien être représentatif?

Le biographe est en présence d'une aporie, d'une impasse résultant, selon Aristote, de "l'égalité des raisonnements contraires", d'une situation où "résonnant dans les deux sens, toutes les raisons nous apparaissent égales de part et d'autre». L'aporie philosophique ou logique est la suivante: que dit Dessaulles de l'Homme en général, que dit l'Homme de Dessaulles en particulier. Cette articulation logique du particulier au général et vice-versa n'est pas résolue philosophiquement. Elle ne l'est pas non plus dans sa formulation sociologique: comment se fait l'arrimage entre l'individu et la société? Que peut dire l'individuel du social ? On peut bien prétendre résoudre cette situation sans issue par des pirouettes de langage - l'homme et son temps, $\mathrm{X}$ comme microcosme de son époque, «la» figure de ceci ou de cela -, mais il me semble que tant que le biographe n'a pas accepté de vivre avec cette aporie, il vit en situation de méprise occultée et de risque de généralisation abusive. Dessaulles n'est donc «la" figure ni du Canadien français ni du libéral du xix ${ }^{\mathrm{e}}$ siècle; au mieux, il représente l'avancée la plus radicale du libéralisme canadienfrançais du $\mathrm{XIX}^{\mathrm{e}}$ siècle et l'on est légitimé de le qualifier ainsi parce que, entre autres bonnes raisons, des libéraux radicaux contemporains comme Joseph Doutre et Arthur Buies l'ont reconnu en tant que tel.

Pareil énoncé - l'évaluation des contemporains - comporte une position épistémologique d'intérêt pour le biographe. Lui qui écrit pour ses propres contemporains doit relever le défi de reproduire son protagoniste sous des traits reconnaissables par ses contemporains, conformes à la représentation que ses contemporains ont d'eux-mêmes. Ce défi me semble être une tension souhaitable dans la rédaction d'une biographie, un principe directeur de recherche et d'écriture.

Ce que j'ai trouvé de moins mauvais, non pour résoudre, mais pour faire face à l'aporie de l'individuel et du social, renvoie à ce que j'ai appelé la spirale de la sociabilité. Faute de pouvoir expliquer les modes d'arrimage de l'individu à la société, j'ai décrit ceux successifs et simultanés, d'insertion de Dessaulles dans la société plus vaste que lui-même, de la famille nucléaire à la famille élargie, à la «famille » collégiale, amicale, idéologique. 


\section{IDENTIFIER DES CAS-PROBLÈMES}

La proposition vient de Jacques Le Goff qui a pratiqué la biographie ${ }^{2}$; elle est sa façon de répondre à la question: quel est l'apport scientifique de la biographie? La biographie n'est pas la plaisante et facile narration d'une vie, la répétition plus ou moins romancée des connaissances sur un destin qu'on peut regarder du point de vue de Sirius. La biographie, le traitement individuel du temps et de la "société », fait voir des aspects, des accents, des excès qui peuvent permettre d'identifier ou d'approfondir un questionnement. Grâce à Dessaulles, il me semble que j'ai identifié quelques cas-problèmes. D'abord, la question de la typologie du libéralisme, des composantes du libéralisme radical et modéré et, surtout, de la position dominante du libéralisme modéré après 1849. L'étude de Dessaulles, du milieu de L'Avenir, du Pays et des Instituts canadiens de Montréal et de Saint-Hyacinthe auquel il appartient, m'a fait clairement voir 1) que l'anticléricalisme constitue la marque différentielle entre les deux libéralismes ; 2) que le renversement en faveur du libéralisme modéré s'opère en 1848 (question romaine, échec de la stratégie de rappel de l'Union) et en 1849 (marginalisation de Papineau, échec de la stratégie annexionniste); et 3) que c'est sur la question anticléricale - des États pontificaux et du pouvoir temporel du pape aussi qu'achoppe alors le projet nationalitaire des Rouges.

J'ai étudié simultanément la vie de Dessaulles et le phénomène de la conférence publique sous l'Union et la Confédération. Il est donc difficile, sinon impossible, de dissocier ces deux objets, mais il m'apparaît évident que c'est Dessaulles qui m'a permis de faire le point - au sens optique du terme - sur la conférence publique. Je découvrais que j'avais affaire au conférencier le plus actif, le plus publié et au cœur de références multiples à d'autres conférenciers.

Ceux qui voudraient prendre en défaut celui qui - ultérieurement ${ }^{3}-$ affirma qu'on ne pouvait employer pour le $x x^{e}$ siècle québécois le substantif «intellectuel» appliqué à des Canadiens français n'auraient qu'à aller à la page 12 de mon Dessaulles: «Je tenais, enfin, un intellectuel acceptable, enviable, qui n'était ni Camus ni Sartre!» Je n’ai pas péché! J'ai simplement entrevu pour la première fois une dimension de

2. Jacques Le Goff, « Comment écrire une biographie historique aujourd’hui?, Le Débat, 54 (mars-avril 1989) : 48-53.

3. Yvan Lamonde, "Les intellectuels francophones au Québec au XIX siècle : questions préalables ", Revue d'histoire de l'Amérique française, 48,2 (automne 1994): 153-185. 
l'intellectuel — la valorisation, l'usage et la défense de la raison - , qui, en sus du non-usage du substantif «intellectuel » au XIX siècle québécois, me légitime d'affirmer qu'il n'y a pas d'intellectuel au Québec au $\mathrm{xIx}^{\mathrm{e}}$ siècle et que son émergence y est fonction de l'évolution du statut social de la raison.

\section{LE RECIT (ICI LE PLAISIR)}

L'historien est habitué à écrire une thèse, un article pour revue spécialisée, une monographie sur ceci ou cela. L'écriture historique est parfois présentée - il est très rare que la formation universitaire du futur historien prenne en considération la dimension de la communication de la recherche - comme une nécessaire mise à plat, comme le rendu d'une réalité, d'un référent objectif fait de dates ou de chiffres. Bref, l'écriture en histoire est un mal nécessaire, le prosaïque moyen d'une tâche plus pressante: «l'objectivité ».

L'historien transformé en biographe fait face dès la première phrase de sa biographie au problème de l'écriture historique: comment conter cette vie? Par où commencer, en suivant quelles trames? Comment faire face aux trous de la connaissance ou de la documentation? Que faire avec le "fil blanc", celui qui sert à coudre des explications plausibles sur l'inconnu, à supposer des choses chez son "protagoniste"? Une fois "le chemin parcouru ", l'idée s'impose au biographe que le "réel» auquel il se fait si fort de référer n'est accessible qu'à travers le langage, qu'à travers un récit qui lui donne sens. L'histoire communiquée, contée est nécessairement narrative et ce n'est pas consentir à la "romance " que de le reconnaitre. L'historien et le biographe font des choix de récit, optent pour des stratégies narratives variées ${ }^{4}$.

La biographie comporte d'entrée de jeu ce défi et ce plaisir. L'historien $\mathrm{y}$ retrouve le récit et un étonnement global face à son travail.

\section{DE L'OBJECTIVATION}

Quel étonnement? Celui, tout simple de se demander ce qui appartient au biographe et ce qui appartient à son personnage. Pourquoi ai-je choisi de faire la biographie d'un personnage polémiste et frontal comme Dessaulles? Par quoi étais-je attiré chez cet individu, chez ce personnage

4. Pour le point sur la question de l'écriture historique : Roger Chartier, «Figures rhétoriques et représentations historiques", dans $A u$ bord de la falaise. L'histoire entre certitudes et inquiétude (Paris, Albin Michel, 1998), 108-125. 
qu'un opposant décrivait comme un don Quichotte sur sa Rossinante? Pourquoi un autre biographe choisit-il Laurier, Asselin, $\mathrm{M}^{\mathrm{gr}}$ Taschereau, $\mathrm{M}^{\mathrm{gr}}$ Laflèche ou Julie Papineau ? Peut-on y avoir quelque forme d'identification ou de contre-identification? Comment prévenir les dérapages possibles de projection de soi sur son personnage? Qu'y a-t-il de révélateur, après coup, dans les exergues que j'ai choisis en tête de chacun des chapitres de mon Dessaulles? Pourquoi avoir tenu compte de cette décantation de Dessaulles par son grand ami Joseph Doutre, qui écrit à Buies, un an après l'exil du polémiste: "Faites bien attention à ceci: quand vous reprochez quelque chose au clergé, et ce sera, hélas, tous les jours, n'impliquez jamais la totalité du clergé. Laissez toujours un noyau d'hommes sages dans lequel pourront se classer ceux qui nous approuveront. Si vous attaquez tout le corps comme le faisait Dessaulles, vous constituez vous-même tout le corps en hostilité déclarée et implacable. Cela ne coûte rien de faire toujours une exception. Au reste une exception existe toujours quelque part. " Le biographe a-t-il appris à propos de Dessaulles ce que des contemporains de même allégeance avaient appris du même homme? En ce sens, la biographie, qui met en cause un humain, a quelque chose de thérapeutique; elle met en branle toute la dimension de sciences "humaines" de l'histoire et remet en interrogation les procédures d'objectivation du travail historique ${ }^{5}$.

\section{ECRIRE POUR QUI?}

En dehors de la fiction, la biographie est le genre «littéraire» le plus apprécié du lecteur moyen et la biographie historique est souvent perçue comme un moyen agréable d'entrer dans une époque, dans une mentalité, dans une expérience individuelle intéressante pour des raisons fort variées. La biographie pose au biographe et à l'historien la question de la communication: pour qui écrit-il? Pour ses pairs, dans le langage plus ou moins codé de la corporation savante? Pour un public plus large d'individus et de citoyens friands de mieux apprécier un passé qui marque d'une façon ou d'une autre leur présent?

La conjoncture de l'enseignement de l'histoire au Québec depuis trois décennies, l'édition historique et la place de l'histoire dans les médias radiophoniques, télévisuels et touristiques indiquent qu'il y a une demande d'histoire. La biographie historique est l'un des meilleurs

5. Yvan Lamonde, "La fiche et le divan. Sur le faire de l'historien", Trans (revue de psychanalyse), 10 (1999): 171-180. 
moyens de prendre en compte cette demande. Imaginons un moment que le lectorat québécois puisse disposer d'une biographie de PierreStanislas Bédard, de Denis-Benjamin Viger, de Louis-Joseph Papineau, de Louis-Hippolyte La Fontaine, de George-Étienne Cartier; qu'il puisse faire l'histoire religieuse du Québec à travers la biographie de $\mathrm{M}^{\mathrm{gr}}$ Plessis, de $\mathrm{M}^{\mathrm{gr}}$ Bourget, de $\mathrm{M}^{\mathrm{gr}}$ Taschereau; qu'il traverse le $\mathrm{xx}^{\mathrm{e}}$ siècle intellectuel en lisant une biographie de Lionel Groulx, d'Henri Bourassa, de Jules Fournier, d'André Laurendeau, de Fernand Dumont. La mémoire serait alors probablement plus vivante et la conscience historique, plus ferme. 